\title{
The Analysis on the Activity of Daily Living of Alzheimer's Disease
}

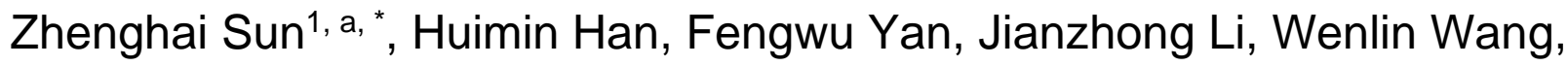 \\ Yunfeng Han, Jingwen Meng and $\mathrm{Na}$ Wang \\ ${ }^{1}$ College of mental health, Qiqihar Medical University, Qiqihar 161006, China. \\ azhsun1980@sina.com
}

\begin{abstract}
Objective: To investigate the influence level of activity of daily living about the patients about the Alzheimer's disease. Methods: Stratified multistage cluster sampling method to determine the survey, investigation and clinical diagnosis using two-stage screening method, screening stage using mini mental-mental state examination (MMSE) and activities of daily living (ADL), to test. According to the diagnostic phase of clinical history, clinical examination, neuropsychological testing to assess, diagnosis using DSM-IV and NINCDS-ADRDA criteria. On AD patients and normal elderly controls were assessed activities of daily living scale (ADL), evaluation and analysis of AD patients' daily living activities. Results: (1) The 3698 survey of 60 -year-old and 60 years of age or older, the identified Alzheimer's disease (AD) patients with 87, Alzheimer's disease (AD) prevalence rate was $2.4 \%$, which accounted for $59.77 \%$ of light; medium $24.14 \%$; heavy accounted for $16.09 \%$.(2) AD patients whose scores of activities of daily living, physical aspects and tools for self-care activities of daily living were significantly higher than normal $(\mathrm{t}=8.02, \mathrm{P}<0.01 ; \mathrm{t}=6.22, \mathrm{P}$ $<0.01 ; \mathrm{t}=8.15, \mathrm{P}<0.01)$, two completely normal activities of daily living, decreased the rate of decline and the difference was statistically significant $(\chi 2=24.09, \mathrm{P}<0.01)$, description of daily living in patients with $\mathrm{AD}$ were lower; physical aspects of daily living score was significantly higher than tools for self-care activities of daily living score $(\mathrm{t}=6.79, \mathrm{P}<0.01)$, showed a more marked decline in physical function; patient groups of different age difference between the mean score of ADL was statistically significant $(\mathrm{F}=5.49, \mathrm{P}<0.01)$, showed ages, worse activities of daily living; patients groups of different severity difference between the mean score of ADL was statistically significant $(\mathrm{F}=44.58, \mathrm{P}<0.01)$, showed the more severe the disease, the more obvious activities of daily living impaired. Conclusion: Alzheimer's disease (AD) seriously affect the ability of daily life, AD patients with activities of daily living, physical aspects and tools for self-care activities of daily living were significantly lower, tools for self-care activities of damage was more obvious; the older, more serious disease, the more serious damage of daily living.
\end{abstract}

Keywords: Alzheimer's disease; prevalence; activity of daily living.

\section{老年性痴呆患者日常生活能力受损情况分析}

\section{孙正海 ${ }^{1}$, 韩惠民，间凤武，李荐中，王文林，韩云峰，孟静文，王娜}

1. 齐齐哈尔医学院精神卫生学院, 齐齐哈尔 中国

摘 要: 目的 探讨老年性痴呆（Alzheimer's disease, AD) 患病对老年人日常生活能力的 影响。方法 采用分层多级整群的抽样方法确定调查对象, 调查采用篮查和临床诊断两阶段 法, 笁查阶段采用简易智能精神状态量表（MMSE）和日常生活能力量表（ADL）进行测验。临 床诊断阶段根据病史、临床体格检查、神经心理测验作出评定, 诊断采用DSM- IV 和 NINCDS-ADRDA的标准。对AD患者和正常对照组老年人进行日常生活能力量表的评定（ADL）， 评价分析 $A D$ 患者的日常生活能力。结果 (1) 调查的3698名60岁及60岁以上的老年人中, 查 出老年性痴呆 $(A D)$ 患者 87 人, 老年性痴呆 $(A D)$ 的患病率为 $2.4 \%$, 其中轻型占 $59.77 \%$; 中 型占24.14\%; 重型占16.09\%。（2）AD患者的日常生活能力、躯体生活自理方面和工具生活自 理方面的评分都显著高于正常人 $(t=8.02, P<0.01 ; t=6.22, P<0.01 ; t=8.15, P<0.01)$, 两组 日常生活能力完全正常、下降和明显下降的比率比较差异有统计学意义 $(x 2=24.09$, 
$P<0.01 ）$, 说明AD患者日常生活能力明显低于正常人; 工具生活自理能力方面评分明显高于 躯体生活自理方面评分（ $t=6.79, P<0.01$ ), 说明工具生活能力下降的更明显; 患者组不同年 龄段之间ADL平均得分比较差异有统计学意义（F=5.49, $P<0.01)$, 说明年龄增大, 日常生活 能力越差; 患者组不同严重程度间ADL平均得分比较差异有统计学意义（F=44. 58, $P<0.01 ）$, 说明疾病越严重, 日常生活能力受损越明显。结论 老年性痴呆（AD）严重影响老年人日常 生活能力, $A D$ 患者的日常生活能力、躯体生活自理方面和工具生活自理方面都显著低于正常 人, 工具生活自理方面受损更明显, 且年龄越大, 疾病越严重, 日常生活能力受损越严重。

\section{关键词: 老年性痴呆; 患病率; 日常生活能力}

\section{1. 前言}

老年性痴呆又称阿尔茨海默病 (Alzheimer's disease, AD) 是神经系统的退行性变所致的 大脑皮质高级精神行为活动的功能障碍, 是获得性、全面性、持续性智能障碍综合征, 是老 年期疾呆的主要类型。AD已成为继心脑血管病、恶性肿瘤之后危害老年人生命的主要疾病之 一, 将给社会经济发展和老年卫生保健带来沉重的负担, 且日益受到世界各国的高度重视 ${ }^{[1,2]}$ 。 中国老年痴呆症患者达 1000 万人, 患病人数已居世界各国之首. 据预测, 到 2050 年, 每 85 人 中就有 1 人罹患老年痴呆症 ${ }^{[3]}$, 我国老年痴呆症患者将激增至 2.8 亿人 ${ }^{[4]}$, 可见防治老年痴 呆症已刻不容缓。本研究通过对AD患者进行神经心理学评估, 以了解 $A D$ 患病对老年人日常生 活能力的影响及损害程度。

\section{2. 对象和方法}

\section{1. 抽样方法和调查对象}

采用分层多级整群方法抽样方法抽取齐齐哈尔市富拉尔基区的 8 个居委会和 1 个村委会中 符合入选条件的所有年龄在 60 岁及以上者作为调查对象, 其入选标准为: 在调查地有常住户 口, 调查时居住在调查地; 在调查地无常住户口, 调查时居住在该地常住户的亲属家已满一 个月者; 调查时迁出不满一个月的常住人口, 对知情者进行问卷调查。参照我国各地关于阿 尔茨海默病的流行病学调查 ${ }^{[5]}$ 结果, 尤其是和富拉尔基地区经济及环境比较相似地区的调查 结果, 按照患病率5\%左右估计样本含量 3500 人, 抽样所得应查人口 3984 人, 实查 3698 人, 由 于各种原因, 失访 286 人, 实查率为 $92.82 \%$ 。失访原因为外出、死亡、拒绝等。

\section{2. 调查方法}

调查采用与国际接轨的两阶段调查法（篮查和临床诊断两阶段）。中国修订版简易智能 精神状态量表 (MMSE) ${ }^{[6]}$ 进行认知功能检查; 严重的认知损害或失语等其他原因, 无法进行 MMSE量表评定时, 知情者认为确有认知障碍的, 可以根据临床表现和日常生活能力量表 (ADL) ${ }^{[6]}$ 评分判断。MMSE异常的判定采用文化调整的划界值, 文盲 $\leqslant 19$ 分, 小学组 $\leqslant 22$ 分, 初中组 $\leqslant$ 26 分 $^{[7]}$ 。临床诊断由临床医生进行系统的病史搜集、一般检查、神经系统检查和精神状态检

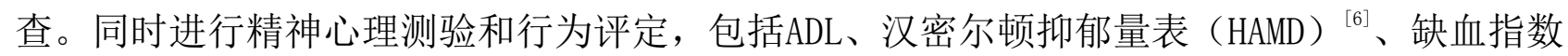
量表 (Hachinski) ${ }^{[6]}$ 。根据病史和测验结果由 2 名以上医生分别独立进行诊断, 两人判定结 果不一致时由第三位医生再次判断, 并由课题组成员组织病例讨论, 同时进行痴呆的诊断、 分型。为了了解 $A D$ 患病对老年人日常生活能力的影响程度, 我们对所有 87 例确诊的 $A D$ 患者采 用日常生活能力量表 (ADL) 进行评定, 并随机选取87例与患病组同社区, 同性别、同文化程 度、年龄相当 $( \pm 3$ 岁 $)$ 的无认知功能障碍和严重的脑血管及其他中枢神经系统疾病的正常老 年人进行日常生活能力量表 (ADL) 评定, 两者进行对照分析。每项按 $1^{\sim}$ 4级标准分级评分, 然后将 20 项评分进行累加, 即为个人的ADL得分值。总分 20 分为正常; 大于 20 分即有不同程度 功能下降; 单项 1 分为正常, $2^{\sim} 4$ 分为功能下降; 总分大于等于 22 分即有明显的功能下降。躯 
体生活自理方面的为第 $2,5,6,8,10,11,12,14,17,20$ 项, 工具生活自理方面的为第 1 , $3,4,7,9,13,15,16,18,19$ 项。

2.3. 诊断标准

痴呆的诊断参照美国精神疾病诊断与统计手册第4版 (DSM-IV) ${ }^{[8]}$ 的标准, AD的诊断参照 美国神经病学、语言障碍和卒中一老年性痴呆和相关疾病学会 (NINCDS-ADRDA) ${ }^{[9]}$ 标准。

2.4. 统计分析

全部资料经统一编码后采用EpiData3. 1软件录入, 用SAS9.13进行资料的统计分析。进行 各项描述性分析、方差分析、 $\mathrm{t}$ 检验、 $\mathrm{x}^{2}$ 检验、趋势性检验。

\section{3. 结果}

一般情况调查的 3698 名 60 岁及 60 岁以上的老年人中，男性 1916 人，女性 1782 人。排除血 管性痴呆和混合性痴呆，共确诊老年性痴呆患病人数共 87 例，老年性痴呆的患病率为 $2.4 \%$ 。 根据MMSE评分情况结合临床表现对87例老年性痴呆患者进行了严重程度分型, 其中轻型患者 52 人，占 $59.77 \%$; 中型患者 21 人，占 $24.14 \%$ ；重型患者 14 人，占 $16.09 \%$ 。

3. 1. 健康组与患者组ADL评分

表1 健康组与痴呆组ADL评分

\begin{tabular}{cccc}
\hline 组别 & ADL平均得分 & 躯体生活自理方面 & 工具生活自理方面 \\
\hline 患者组 & $36.78 \pm 16.97$ & $15.98 \pm 8.04$ & $20.43 \pm 9.80$ \\
健康组 & $21.92 \pm 2.69$ & $10.43 \pm 0.84$ & $11.49 \pm 2.25$ \\
$\mathrm{t}$ 值 & 8.02 & 6.22 & 8.15 \\
$\mathrm{P}$ 值 & $\mathrm{P}<0.01$ & $\mathrm{P}<0.01$ & $\mathrm{P}<0.01$ \\
\hline
\end{tabular}

表2 $\mathrm{AD}$ 患者组和健康组日常生活能力得分情况

\begin{tabular}{ccc}
\hline 项目 & AD患者组ADL平均得分 & 健康组ADL平均得分 \\
\hline 1. 自己搭公共汽车 & $2.13 \pm 1.24$ & $1.24 \pm 0.43$ \\
2. 到家附近的地方去 (步 & $1.78 \pm 1.13$ & $1.02 \pm 0.21$ \\
行范围) & $2.18 \pm 1.29$ & $1.20 \pm 0.49$ \\
3. 自己做饭 (包括生火) & $1.48 \pm 0.84$ & $1.04 \pm 0.34$ \\
4. 吃药 & $1.42 \pm 0.80$ & $1.01 \pm 0.11$ \\
5. 吃饭 & $1.60 \pm 1.05$ & $1.02 \pm 0.21$ \\
6. 穿衣服、脱衣服 & $2.09 \pm 1.25$ & $1.07 \pm 0.30$ \\
7. 做家务 & $1.67 \pm 1.05$ & $1.06 \pm 0.28$ \\
8. 梳头、刷牙等 & $1.91 \pm 1.11$ & $1.17 \pm 0.46$ \\
9. 洗自己的衣服 & $1.40 \pm 0.79$ & $1.01 \pm 0.11$ \\
10. 在平坦的室内走 & $1.58 \pm 0.97$ & $1.01 \pm 0.11$ \\
11.上下楼梯 & $1.55 \pm 0.98$ & $1.02 \pm 0.15$ \\
12. 上下床、坐下或站起 & $2.23 \pm 1.27$ & $1.24 \pm 0.46$ \\
13. 提水者饭 & $2.13 \pm 1.19$ & $1.25 \pm 0.49$ \\
14. 洗澡 & $2.00 \pm 1.23$ & $1.15 \pm 0.36$ \\
15. 剪脚趾甲 & $2.31 \pm 1.31$ & $1.23 \pm 0.45$ \\
16. 逛街、购物 & $1.65 \pm 1.01$ & $1.00 \pm 0.00$ \\
17. 定时去则所 & $2.13 \pm 1.29$ & $1.03 \pm 0.18$ \\
18. 打电话 & $2.08 \pm 1.26$ & $1.10 \pm 0.34$ \\
19. 处理自己的钱财 & $1.60 \pm 1.02$ & $1.01 \pm 0.11$ \\
20. 独自在家 & &
\end{tabular}


患者组ADL平均得分 $36.78 \pm 16.97$, 躯体生活自理方面 $15.98 \pm 8.04$, 工具生活自理方面 $20.43 \pm 9.80$; 健康组ADL平均得分 $21.92 \pm 2.69$, 躯体生活自理方面 $10.43 \pm 0.84$, 工具生活自 理方面 $11.49 \pm 2.25$, 提示老年性痴呆组日常生活能力均较健康组差, 经统计学检验差别成显 著性（见表1）。患者组工具生活自理方面较躯体生活自理方面下降明显, 主要以工具生活能 力下降为主, 经统计学检验差别成显著性（ $t=6.79, \mathrm{P}<0.01)$ （各方面评分结果见表2）

3.2. 对 $A D$ 患者组和健康组个人ADL评分

$\mathrm{AD}$ 患者组中 $20.69 \%$ 的 $\mathrm{AD}$ 患者日常生活能力完全正常, 79.31\%的AD患者日常生活能力下降, $75.86 \%$ 的AD患者日常生活能力明显下降; 健康组中 $52.87 \%$ 的健康者日常生活能力完全正常, 47. 13\%的健康者日常生活能力下降, 39.08\%的健康组日常生活能力明显下降。两组比较差异 有统计学意义 $\left(x^{2}=24.09, P<0.01\right)$ 。

3.3. 患者组不同年龄段ADL评分比较

经方差分析，患者组不同年龄段之间 ADL平均得分比较差异有统计学意义 $(\mathrm{F}=5.49$, $\mathrm{P}<0.01)$, 躯体生活自理方面比较差异有统计学意义（ $\mathrm{F}=4.78, \mathrm{P}<0.01)$ ，工具生活自理方面 比较差异有统计学意义 $(\mathrm{F}=5.10, \mathrm{P}<0.01)$ 。

3. 4. 患者组不同性别间ADL评分比较

患者组男性ADL平均得分 $40.23 \pm 17.84$, 躯体生活自理方面 $17.52 \pm 8.43$, 工具生活自理方 面22.12士10.47; 女性ADL平均得分 $34.41 \pm 16.10$, 躯体生活自理方面 $14.94 \pm 7.68$, 工具生活 自理方面19.33土9.28。两组经统计学检验差别无统计学意义（见表3）

表3 患者组不同性别间ADL评分比较

\begin{tabular}{cccc}
\hline 组别 & ADL平均得分 & 躯体生活自理方面 & 工具生活自理方面 \\
\hline 男性 & $40.23 \pm 17.84$ & $17.52 \pm 8.43$ & $22.12 \pm 10.47$ \\
女性 & $34.41 \pm 16.10$ & $14.94 \pm 7.68$ & $14.94 \pm 7.68$ \\
$\mathrm{t}$ 值 & 1.57 & 1.43 & 1.28 \\
$\mathrm{P}$ 值 & $\mathrm{P}>0.05$ & $\mathrm{P}>0.05$ & $\mathrm{P}>0.05$ \\
\hline
\end{tabular}

3.5. 患者组不同严重程度之间ADL评分比较

经方差分析，患者组不同严重程度间 ADL平均得分比较差异有统计学意义 $(\mathrm{F}=44.58, \mathrm{P}<0.01)$ 。

\section{4. 讨论}

本研究发现，健康组ADL总分、工具生活自理方面得分、躯体生活自理方面得分均明显低 于痴呆组, 二者之间存在显著性的差异, 说明痴呆组患者日常生活能力显著低于正常人。 $\mathrm{AD}$ 患者组中 $20.69 \%$ 的AD患者日常生活能力完全正常, 79.31\%的AD患者日常生活能力下降, 75.86\% 的 $\mathrm{AD}$ 患者日常生活能力明显下降，与健康组比较差异显著，且工具生活自理方面较躯体生活 自理方面下降明显, 说明该病对老年人日常生活能力影响严重, 本结果与张明园等研究结果相 一致 ${ }^{(10)}$ 。痴呆患者的日常生活能力缺陷与认知障碍密切相关 ${ }^{(11)}$ ，认知过程（包括注意、记 忆、定向始动性和计划性) 明显受累, 其高级脑功能受累, 出现失用、失认等功能障碍均会导 致日常生活能力的降低。患者组不同严重程度间ADL评分差异明显, 说明随着老年性痴呆疾病 的加重，患者日常生活能力受损也更明显。患者组不同年龄段ADL总分、工具生活自理方面和 躯体生活自理方面比较有显著差异, 提示ADL评分与样本年龄密切相关, 年龄越大, 日常生活 能力受损越严重, 本结果与吕桦等研究结果相一致。这可能与随着年龄升高患慢性疾病的几 率升高及家庭结构受损越易发生等有关。本研究未发现, ADL评分与性别和文化程度明显相关, 与何燕玲等研究结果一致 ${ }^{(12)}$ 。 


\section{致谢}

黑龙江省卫生厅项目黑龙江省齐齐哈尔城区老年性痴呆流行病学研究（2012-344）

\section{References}

[1] Qi Jinshun,Wang Hongqi,Qiao Jiantian.Why the Alzheimer's disease become the focus of the modern medicine multidisciplinary [J]. Medicine and Philosophy, 1999, 21 (7): 47-49.

[2] Qi Bin. Alzheimer's disease rehabilitation nursing[J].Chinese medical innovations, 2010, 7 (23):144-145.

[3] Yu Dalin,Xiao Jun.Epidemiological investigation of Alzheimer's disease [ J ] .Practical Journal of Clinical Medicine,2011,8 (3): 152-155.

[4] HENEKA M T,KUMMER M P,STUTZ A,et al.NLRP3 is activated in Alzheimer's disease and contributes to pathology in APP/PSI mice [J]. Nature,2013,493(7434):674-678.

[5] Wang HuaLi,Yu Xin.The epidemiological situation of Alzheimer's disease in china[J].Chinese Journal of General Practitioners, 2006;5:358-360.

[6] Xu Xianhao,Peng Dantao.Neuropsychological scale testing guidelines[M].BeiJing:China union medical university pree. 2007,10.

[7] Zhang Zhenxin,Hong Xia,Li Hui,etc.Beijing urban and rural residents 55 years of age or over simple distribution characteristics of the test about MMSE[J]. Chinese Journal of Neurology, 1999;32: 149-153.

[8] American Psychiatric Association Diagnostic and statistical of mental disorders .4th ed [M].Washington DC: American Psychiatric Association.1994:143-147.

[9] Mckhann G.Drachmate D.Folstein M.et al Clinical diagnosis of Alzheimer's disease: report of the NINCDS-ADRDA Work Group under the auspices of Department of Heath and Human Services Task Force on Alzheimer's Disease[J].Neurology.1984.34(7):939-944.

[10] Zhang Mingyuan,Zhu Ziqing,Chen Peijun,etc.The elderly daily life ability and some disease of social investigation[J].Chinese medical journal, 1998;78(2):124-127.

[11] Eisdorfer c,Cohen D,Paveza G,etal.An empirical evaluation of the global deterioration scale for staging Alzheimer's disease.Am J psychiatry,1992;149:190-194.PMID:1734738

[12] He Yanling,Zhang Mingyuan,Li Roubing.The change of the elderly daily life ability and its relationship with dementia[J].Chinese Journal of Clinical Psychology, 1998;6(1)20-23. 